\title{
Joint mobilization associated or not to other therapies reduces chronic musculoskeletal pain: a systematic review
}

\author{
Mobilização articular associada ou não a outras terapias reduz dor musculoesquelética crônica: \\ uma revisão sistemática
}

Thiago dos Santos Sousa Abner ${ }^{1}$, Maria Ivone Oliveira Dantas², Isabela Freire Azevedo-Santos ${ }^{3}$, Josimari Melo DeSantana',2

DOI 10.5935/2595-0118.20200015

\section{ABSTRACT}

BACKGROUND AND OBJECTIVES: Joint mobilization is a non-pharmacological technique used to treat chronic musculoskeletal pain. However, it is controversial due to a lack of studies comparing its effects on this painful condition. The objective of this study was to assess the risk of bias in clinical trials investigating the effect of joint mobilization on chronic musculoskeletal pain.

CONTENTS: A systematic search on Pubmed, Cochrane Library, ScienceDirect, Scielo, PEDro, CINAHL, SPORTDiscus, LILACS, BVS, PsycINFO, Web of Science, and Scopus was performed on September 2019 from the combination of three keywords: Musculoskeletal Manipulations AND Chronic Pain AND Musculoskeletal Pain. Randomized controlled clinical trials that evaluated the use of joint mobilization associated or not to other therapies in chronic musculoskeletal pain treatment were included. Five thousand five hundred eighty-seven articles were screened, and 14 studies were analyzed, including 812 participants, with a mean age of 54 years, and female being the most affected. According to these articles, joint mobilization promoted the reduction of pain intensity in short and long terms, increase in range of motion, strength and function when used alone or in association with conventional physiotherapy. Regarding methodological quality, most of the studies were classified with low risk for selection, performance, detection and reporting bias. In the "other bias" item, which considered therapists experience

Thiago Abner dos Santos Sousa - (Dhttps://orcid.org/0000-0001-9653-3116; Maria Ivone Oliveira Dantas - Dhttps://orcid.org/0000-0003-0588-4901;

Isabela Azevedo Freire Santos - Dhttps://orcid.org/0000-0001-8836-8640;

Josimari Melo DeSantana - (Dhttps://orcid.org/0000-0003-1432-0737.

1. Universidade Federal de Sergipe, Programa de Pós-Graduaçăo em Ciências da Saúde, Aracaju, SE, Brasil.

2. Universidade Federal de Sergipe, Programa de Pós-Graduação em Ciências Fisiológicas, Aracaju, SE, Brasil.

3. Centro Universitário Estácio de Sergipe, Curso de Fisioterapia, Aracaju, SE, Brasil.

Submitted on November 19, 2019.

Accepted for publication on January 02, 2020.

Conflict of interests: none - Sponsoring sources: none.

Correspondence to:

Departamento de Fisioterapia, Universidade Federal de Sergipe

Av. Marechal Rondon, s/n, Jardim Rosa Elze

49100-000 São Cristóvão, SE, Brasil.

E-mail: desantanajm@gmail.com

(C) Sociedade Brasileira para o Estudo da Dor time and types of treatment applied, only one study presented low risk and other study presented an unclear risk.

CONCLUSION: Joint mobilization seems to be an effective technique for the treatment of chronic musculoskeletal pain. However, it is still necessary to investigate and compile studies with greater methodological quality, thus promoting greater support to evidence-based practice.

Keywords: Chronic pain, Musculoskeletal manipulations, Musculoskeletal pain.

\section{RESUMO}

JUSTIFICATIVA E OBJETIVOS: Mobilização articular é uma técnica não farmacológica usada no tratamento da dor musculoesquelética crônica. No entanto, é controverso devido à falta de estudos que comparem seus efeitos sobre essa condição de dor. O objetivo deste estudo foi avaliar o risco de viés em ensaios clínicos que investigam o efeito da mobilização articular na dor musculoesquelética crônica.

CONTEÚDO: Foi realizada uma busca sistematizada no $\mathrm{Pu}$ bmed, Cochrane Library, ScienceDirect, Scielo, PEDro, CINAHL, SPORTDiscus, LILACS, BVS, PsycINFO, Web of Science e Scopus em setembro de 2019 com a combinação de três palavras-chave: Musculoskeletal Manipulations AND Chronic Pain AND Musculoskeletal Pain. Ensaios clínicos controlados e aleatorizados que avaliaram o uso de mobilização articular associada ou não a outras terapias no tratamento da dor musculoesquelética crônica foram incluídos. Foram encontrados 5587 artigos e analisados 14 estudos, incluindo 812 participantes, com idade média de 54 anos, sendo o sexo feminino o mais afetado. Nestes, a mobilização articular promoveu redução da dor em curto e longo prazo, amplitude de movimento, força e melhora da função quando utilizado isoladamente ou em associação à fisioterapia convencional. Em relação à qualidade metodológica, a maioria dos estudos foi classificada com baixo risco para seleção, desempenho, detecção e viés de relato. No item "other bias", que considerou terapeutas com tempo de experiência e tipos de tratamento aplicados, apenas um estudo apresentou baixo risco e outro estudo apresentou risco incerto.

CONCLUSÁO: Mobilização articular parece ser uma técnica eficaz para o tratamento da dor musculoesquelética crônica. No entanto, é necessário realizar estudos com maior qualidade metodológica, promovendo maior apoio à prática baseada em evidências. Descritores: Dor crônica, Dor musculoesquelética, Manipulaçôes musculoesqueléticas. 


\section{INTRODUCTION}

Chronic musculoskeletal pain (CMP) is defined as a painful condition associated with dysfunction in muscles, ligaments, tendons, bones, and/or adjacent structures that lasts for more than three months ${ }^{1,2}$. Musculoskeletal conditions are the main cause of disability worldwide, with a prevalence ranging from 13.5 to $47 \%$ of the general population ${ }^{3}$. The main risk factors for this condition are advanced age, female sex, smoking, low schooling, sedentary lifestyle, poor social interaction, depression and anxiety ${ }^{4}$.

This type of pain has a multifactorial etiology, which may have a definite cause (traumatic, ischemic, tumor, inflammatory, overload, overuse) or non-specific causes ${ }^{5,6}$. Several characteristics may be present in these patients, as generalized, diffuse and/or local muscle pain, physical and mental fatigue, a decrease of muscle strength, demotivation, sleep disorders, among others ${ }^{6-8}$, which may lead to increased health costs and reduced quality of life?

The treatment of CMP can be performed by a multiprofessional team and consists of pharmacological and/or non-pharmacological therapies ${ }^{10}$. The most used drugs are analgesics, non-steroidal anti-inflammatories, antidepressants, neuroleptics, anticonvulsants and myorelaxants ${ }^{11}$. Some non-pharmacological therapies involve physiotherapeutic techniques such as acupuncture, kinesiotherapy, electrotherapy, thermotherapy, phototherapy, spinal manipulation and massage therapy which aim to reduce pain and improve the quality of life of these patients ${ }^{2,12}$.

Currently, these non-pharmacological treatments are being prioritized because of the lower risk of adverse effects. Manual therapy (MT) is one of these non-pharmacological techniques most widely used in the treatment of dysfunctions affecting the musculoskeletal system. It consists of a physiotherapeutic treatment that includes therapeutic massage, joint mobilization (JM), manipulation, among others ${ }^{2}$.

$\mathrm{JM}$ is a technique used to treat musculoskeletal pain in the axial and appendicular skeletons. It is indicated to reduce pain, muscle spasms, reversible joint hypomobility, positioning/subluxation failure, progressive limitation, and functional immobility ${ }^{13}$. However, this technique has limitations and/or contraindications that consist of irreversible hypomobility, joint effusion and inflammation. The neurophysiological and mechanical effects caused by the use of joint mobilizations provide analgesia in patients with $\mathrm{CMP}^{13,14}$.

Studies suggest ${ }^{13,15,16}$ that JM activates the dorsal area of periaqueductal gray matter (PAG) of the brain, and this influences on pain perception. Studies show an immediate reduction of pain and an increase in sympathetic nervous system activity, suggesting an indirect relationship with the dorsal area of PAG and association between increased stimulation of sympathetic nervous system and reduction of mechanical pain threshold ${ }^{13,17-19}$. An experimental study has shown an analgesic response accompanied by sympathetic nervous system activation after the electrical stimulation of the midbrain, originating in $\mathrm{PAG}^{20}$. Other clinical studies have shown a sympathetic-excitatory change combined with a hypoalgesic response after spinal $\mathrm{JM}$, with increased skin conductance ${ }^{21-29}$, respiratory and heart rate $^{30}$, and decreased skin temperature ${ }^{31,32}$. Changes in central sympathetic activity may be directly related to modulation response during therapeutic intervention ${ }^{29}$ and it is also hypothesized that JM initiates the inhibitory mechanisms in the dorsal region of the $\mathrm{PAG}^{13}$.

Using grade III JM in an experimental model of ankle joint inflammation, showed action through spinal blockade of serotonergic (5HT1) receptors, found in nucleus raphe magnus (NRM) and noradrenergic (alpha 2), located mainly in a small nucleus in the gray matter of the pons, the locus coeruleus. However, blockade of GABA or opioid receptors had no influence on the analgesic effect produced by joint mobilization ${ }^{33}$. These data may suggest that MA reduces CNS pain through non-opioid descending inhibitory pathways from the rostral ventromedial medulla and dorsolateral pontine tegmentum. More recently, the role of the nociceptive adenosinergic system has been shown to mediate the antihyperalgesic effect of MA by activating A1 adenosinergic receptors that predominantly mediate the effects of synaptic transmission in the superficial region of the dorsal horn ${ }^{34}$.

There are several studies on the use of MT in diseases and other conditions, such as chronic spinal pain and osteoarthritis $(\mathrm{OA})^{10,13}$. A recently published meta-analysis about manipulation and mobilization, specifically for the treatment of chronic low back pain, has shown that both therapies appear to be safe and that there is moderate quality in the studies that support the use of these techniques to reduce this type of pain ${ }^{14}$.

Another meta-analysis addressed the use of manual therapy, exercise therapy (ET), or combined treatment for adults with cervicalgia. Quality of included studies was moderate and the authors concluded that combined treatment consisting of MT and ET does not appear to be more effective in reducing the intensity of resting neck pain, cervical spine disability, or quality of life improvement in adult patients with cervicalgia when compared to only $\mathrm{ET}^{35}$. Although it presents moderate scientific evidence according to the previously published studies, the use of JM in patients with CMP still presents controversies due to the lack of studies that directly compare its effects in this painful condition. Therefore, there is an even greater need for studies with methodological quality that is rigorous enough to indicate treatments in this area. Thus, in order to verify the existence of clinical trials related to this topic, this systematic review aimed to investigate and evaluate the effect of protocols for JM application associated or not to other therapies in the treatment of pain and motor performance in patients with CMP.

\section{CONTENTS}

As a PICO strategy, randomized trials with a control or placebo group that evaluated the use of JM associated or not with other therapies in CMP treatment were included in this review. The studies that presented participants older than 18 years old with chronic pain related to musculoskeletal dysfunction for time $\geq 3$ months and who were treated with JM associated or not with other therapies were selected.

Clinical trials comparing any type of JM with placebo or sham intervention, with no other type of treatment, mobilization as an isolated therapy or in combination with other conservative thera- 
pies have been included. Clinical trials comparing different protocols of JM (e.g., different degrees, series, repetitions, and/or body sites/segments) were also included.

The exclusion criteria were studies with participants who presented oncological pain, headache, temporomandibular dysfunction (TMD), other painful conditions. It was also excluded studies with patients who were undergone to other modalities of therapies and/or mobilization under anesthesia or performed by machines as forms of treatment, use of JM only outside the site of pain, studies that did not report how long considered the pain condition as chronic and cross-over clinical trials. Studies that had no full-text accessible, and that was not possible to contact the authors, were also excluded.

The primary outcomes evaluated were pain measured by a validated pain score scale, such as the visual analog scale (VAS) and numerical rating scale (NRS) and pressure pain threshold (PPT), measured by digital pressure algometer. As secondary outcomes were considered: the range of motion data (ROM) accessed through universal goniometer or inclinometer, muscle strength measured indirectly or directly through the isokinetic dynamometer and manual tests, functionality measured by validated functional tests, quality of life through validated questionnaires, such as SF-36 and QoL for general measures, adherence to treatment measured by the number of sessions that the individual performed and patient expectation /satisfaction measured through the patient's report and the Likert scale.

\section{Protocol and register}

This research protocol was registered in the International Registry of Systematic Reviews PROSPERO (CRD 42016046029). The inclusion criteria and analyses of studies were performed according to Preferred Reporting Items for Systematic Reviews and Meta-Analyses (PRISMA) and the Cochrane Handbook for Systematic Reviews of Interventions.

\section{Search strategy}

The studies were screened in the following electronic databases: Pubmed, Cochrane Library, ScienceDirect, Scielo, PEDro, CINAHL, SPORTDiscus, LILACS, BVS, PsycINFO, Web of Science, Scopus and Google Scholar. There were no restrictions on language or year of publication. The search was performed in September 2019 by combiningthefollowingdescriptors:Manual Therapy/Musculoskeletal Manipulations ("Musculoskeletal Manipulations"[mesh terms] OR (manipulations, musculoskeletal) OR (manual therapies) OR (manual therapy) OR (therapies, manual) OR (therapy, manual) OR (manipulation therapy) OR (manipulation therapies) OR (therapies, manipulation) OR (manipulative therapies) OR (manipulative therapy) OR (therapies, manipulative) OR (therapy, manipulative) OR (therapy, manipulation)), Chronic Pain ("Chronic Pain"[mesh terms] OR (Chronic Pains) OR (Pains, Chronic) OR (Pain, Chronic) OR (Widespread Chronic Pain) OR (Chronic Pain, Widespread) OR (Chronic Pains, Widespread); (Pain, Widespread Chronic) OR (Pains, Widespread Chronic) OR (Widespread Chronic Pains)) e Musculoskeletal Pain ("Musculoskeletal Pain"[mesh terms] OR (Musculoskeletal Pains) OR (Pain, Musculoskeletal) OR (Pains, Musculoskeletal)).
Searches were remade immediately before the final analyses and additional studies were retrieved for inclusion. The reference lists of all primary studies were checked, and all articles were revised for additional references. Data collection and analyses were performed in accordance with the methods set out in the Preferred Reporting Items for Systematic Reviews and Meta-Analyses (PRISMA) and the Cochrane Handbook for Systematic Reviews of Interventions.

\section{Data selection and extraction}

Two authors independently extracted data from all studies included using an Excel spreadsheet. This worksheet included extracting information about characteristics of studies, participants, methodological aspects, interventions, comparisons, primary and secondary outcomes, results, chronic pain condition, mobilization type, and association with other interventions. At this stage of the study, disagreements among reviewers were discussed with a third investigator.

\section{Quality assessment}

The risk of bias was assessed using the Cochrane Collaboration tool by two reviewers independently. Thus, six domains were evaluated: selection bias (random sequence generation and allocation concealment), performance bias (participants blinding), detection bias (evaluators blinding), attrition bias (results with incomplete data), reporting bias (selective reporting of outcomes) and other biases. For the judgment of this last type of bias (Other bias) the following aspects were considered: the number of physiotherapists who applied the protocols and their years of experience in the area (over one year), mobilization type (with different characteristics and/or combination with other therapies, presence of control group), no validated placebo for mobilization; compared with different therapies and with different application objectives.

Each of these biases was classified as low risk, high risk, or unclear risk. Review Manager 5.3 was used for all quantitative analyses. The searches were remade immediately before the final analyses in September 2019 and additional studies were retrieved for inclusion in order to ensure the selection of the largest possible number of studies.

\section{Included studies}

The database search recovered 5587 potentially relevant references: Pubmed (258), PEDro (28), CINAHL (32), Cochrane Library (39), LILACS (0), Scielo (2), ScienceDirect (4412), Scopus (343), SPORTDiscus (19), Web of Science (145), PsycINFO (15), BVS (194) e Google Scholar (100). The search retrieved 5587 records of trials after removal duplicates, of which 30 articles were selected for full-text evaluation and 11 clinical trials met the inclusion criteria. Hand search on the reference lists of all primary studies was performed and further three clinical trials were selected; thus, 14 clinical trials were included for qualitative synthesis. Figure 1 shows the flowchart of the search and selection process in this review.

Included clinical trials examining the JM intervention associated or not with other therapies in the treatment of CMP were publi- 


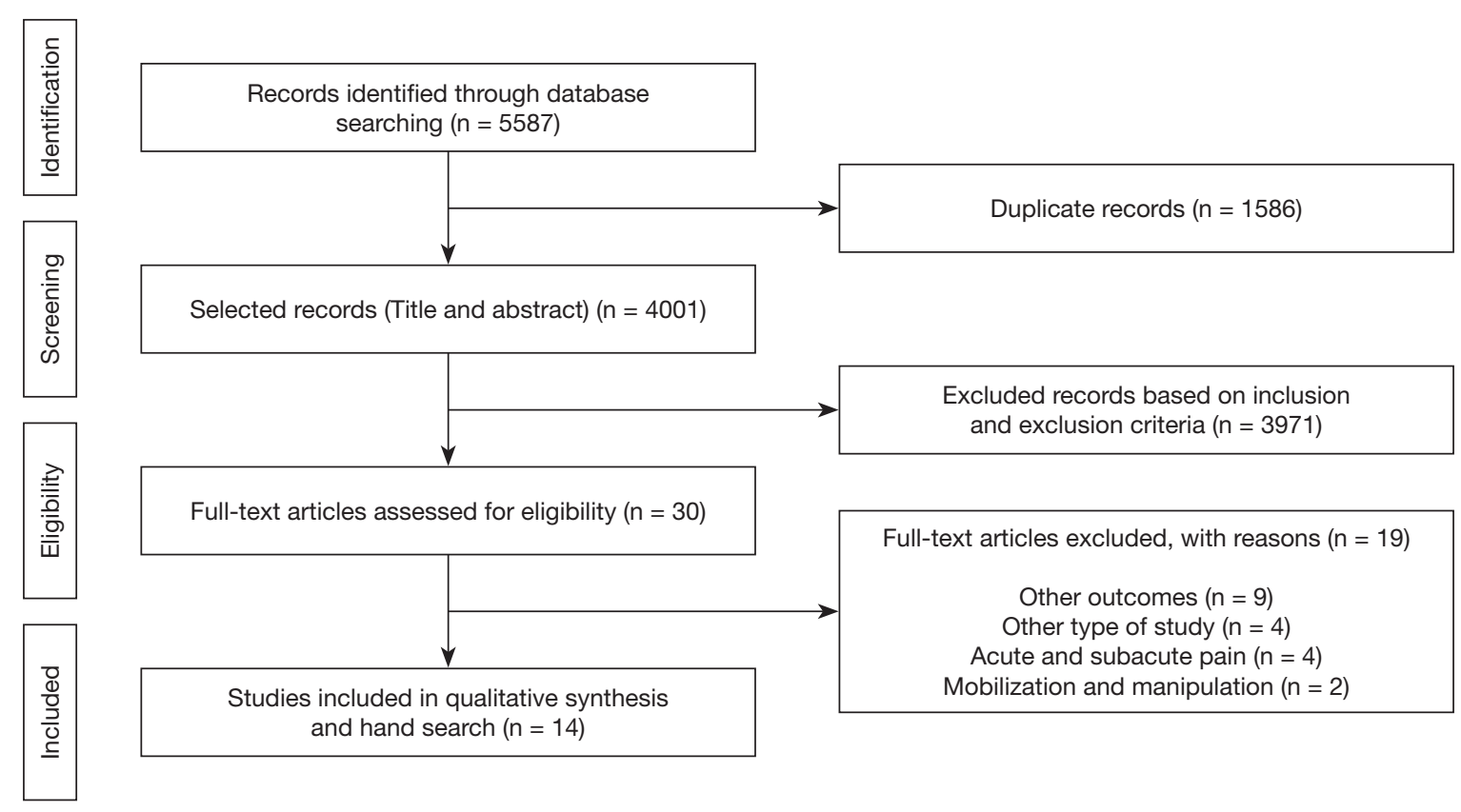

Figure 1. Flow of the studies through the review

shed between 2008 and 2018. The total sample from 14 studies was 812 participants, ranging from 28 to 120 participants in each study. Of these, eleven $(n=11)$ performed the sample calculation to estimate the number of subjects included ${ }^{36-46}$. The mean age of participants was approximately 54 years, ranging from 18 to 90 years. On average, a higher number of female participants were found $(60,98 \%)$.
Some studies $(25 \%)$ considered chronic pain after three months. Of these, two were about cervicalgias ${ }^{41,42}$ and one about rotator cuff injuries $^{36}$. Two studies $(16,66 \%)$ had as inclusion criteria patients with pain for more than 10 years ${ }^{44,47}$. In another study $(8,33 \%)$, chronic epicondylitis as of six months were considered for inclusion ${ }^{15}$. Table 1 summarizes the characteristics of the included studies.

Table 1. Characteristics of the included studies

\begin{tabular}{|c|c|c|c|c|c|c|c|c|}
\hline Authors & Sample & $\begin{array}{l}\text { Chronic pain } \\
\text { condition }\end{array}$ & $\begin{array}{l}\text { Interventions } \\
\text { description }\end{array}$ & $\begin{array}{l}\text { Control } \\
\text { Description }\end{array}$ & Outcomes & Instruments & Results & Conclusion \\
\hline $\begin{array}{l}\text { Bennell } \\
\text { et al. }{ }^{36}\end{array}$ & $\begin{array}{l}\text { Total }(n=120) \\
\text { (experimental } \\
\text { group - EG, } \\
(n=59) \text {, and } \\
\text { control group } \\
\text { - CG ( } n=61) \text {; } \\
22 \text { weeks } \\
\text { follow-up } \\
\text { (EG } n=59 \\
\text { and CG } \\
n=61)\end{array}$ & $\begin{array}{l}\text { Chronic injury } \\
\text { of the rotator } \\
\text { cuff }\end{array}$ & $\begin{array}{l}\text { Soft tissue } \\
\text { massage; } \\
\text { glenohumeral } \\
\text { JM (ante- } \\
\text { roposterior } \\
\text { and inferior } \\
\text { sliding); } \\
\text { Thoracic } \\
\text { and cervical } \\
\text { mobilization } \\
\text { (grade IV); } \\
\text { scapular } \\
\text { rehabilitation; } \\
\text { postural } \\
\text { taping } \\
\text { shoulder } \\
\text { and scapula; } \\
\text { home } \\
\text { exercises }\end{array}$ & $\begin{array}{l}\text { Placebo } \\
\text { ultrasound, } \\
\text { light } \\
\text { application } \\
\text { of non- } \\
\text { therapeutic gel } \\
\text { on the shoulder }\end{array}$ & $\begin{array}{l}\text { Shoulder } \\
\text { pain and } \\
\text { disability; } \\
\text { pain intensity } \\
\text { at rest and } \\
\text { movement; } \\
\text { global } \\
\text { perception; } \\
\text { quality of } \\
\text { life; shoulder } \\
\text { isometric } \\
\text { strength; } \\
\text { adherence to } \\
\text { treatment }\end{array}$ & $\begin{array}{l}\text { SPADI; } \\
\text { NS; } \\
\text { Likert Scale; } \\
\text { SF-36 e AQoL; } \\
\text { Nicholas } \\
\text { Manual } \\
\text { Muscle tester; } \\
\text { Records of } \\
\text { the number } \\
\text { of physical } \\
\text { therapy visits }\end{array}$ & $\begin{array}{l}\text { There was no differen- } \\
\text { ce between groups on } \\
\text { pain and disability of } \\
\text { shoulder, on pain at } \\
\text { rest and movement, } \\
\text { both groups showed } \\
\text { significant improve- } \\
\text { ment; the participants } \\
\text { in active group showed } \\
\text { greater satisfaction } \\
\text { with treatment, despite } \\
\text { non-significant diffe- } \\
\text { rence between groups. } \\
\text { The active group } \\
\text { showed a significant } \\
\text { improvement in SPADI } \\
\text { than placebo group af- } \\
\text { ter } 22 \text { weeks, although } \\
\text { there was no differen- } \\
\text { ce between groups } \\
\text { for pain reduction or } \\
\text { percentage of parti- } \\
\text { cipants who reported } \\
\text { treatment success. The } \\
\text { active group obtained } \\
\text { better muscle strength, } \\
\text { less interference in } \\
\text { activities and better } \\
\text { quality of life }\end{array}$ & $\begin{array}{l}\text { A standardized } \\
\text { program of manual } \\
\text { therapy and home } \\
\text { exercises did not } \\
\text { present immediate } \\
\text { benefits for pain } \\
\text { and function } \\
\text { compared to a } \\
\text { placebo group. } \\
\text { However, greater } \\
\text { improvements } \\
\text { were observed in } \\
\text { shoulder function } \\
\text { and strength at } \\
22-\text { week follow-up, } \\
\text { suggesting that } \\
\text { benefits with active } \\
\text { treatment take time } \\
\text { to manifest }\end{array}$ \\
\hline
\end{tabular}


Table 1. Characteristics of the included studies - continuation

\begin{tabular}{|c|c|c|c|c|c|c|c|c|}
\hline Authors & Sample & $\begin{array}{l}\text { Chronic pain } \\
\text { condition }\end{array}$ & $\begin{array}{l}\text { Interventions } \\
\text { description }\end{array}$ & $\begin{array}{l}\text { Control } \\
\text { Description }\end{array}$ & Outcomes & Instruments & Results & Conclusion \\
\hline $\begin{array}{l}\text { Beselga } \\
\text { et al. } .^{38}\end{array}$ & $\begin{array}{l}\text { Total }(n=40) \\
E G n=20 \text { and } \\
\text { CG } n=20\end{array}$ & Hip OA & $\begin{array}{l}\text { JM with flexion } \\
\text { and internal } \\
\text { hip rotation } \\
\text { (Mulligan } \\
\text { technique) }\end{array}$ & $\begin{array}{l}\text { Simulated } \\
\text { therapy of } \\
\text { mobilization } \\
\text { technique with } \\
\text { hip movement }\end{array}$ & $\begin{array}{l}\text { Pain intensity } \\
\text { at rest; } \\
\text { ROM of } \\
\text { hip flexion } \\
\text { and internal } \\
\text { rotation; } \\
\text { functionality. }\end{array}$ & $\begin{array}{l}\text { NS; } \\
\text { Universal } \\
\text { goniometer; } \\
\text { Timed up and } \\
\text { go test; } \\
\text { 30s Chair } \\
\text { Stand; SPWT }\end{array}$ & $\begin{array}{l}\text { In the EG, there } \\
\text { was pain intensity } \\
\text { reduction, increased } \\
\text { hip flexion and } \\
\text { internal rotation, } \\
\text { and functional tests } \\
\text { also improved with } \\
\text { a relevant clinical } \\
\text { effect. There were no } \\
\text { significant changes in } \\
\text { any outcome in CG }\end{array}$ & $\begin{array}{l}\text { Pain intensity, hip } \\
\text { flexion ROM and } \\
\text { physical performance } \\
\text { improve immediately } \\
\text { after the application } \\
\text { of JM with movement } \\
\text { in patients with hip } \\
\text { OA. The immediate } \\
\text { changes observed } \\
\text { were clinically } \\
\text { relevant. }\end{array}$ \\
\hline $\begin{array}{l}\text { Crossley } \\
\text { et al. }{ }^{39}\end{array}$ & $\begin{array}{l}\text { Total }(n=92) \\
\text { (experimental } \\
\text { group EG } \\
\text { ( } n=39) \text { and } \\
\text { CG }(n=42) ; \\
9 \text { months } \\
\text { follow-up EG } \\
(n=35) \text { and } \\
\text { CG }(n=34))\end{array}$ & $\begin{array}{l}\text { Patellofemoral } \\
\text { OA }\end{array}$ & $\begin{array}{l}\text { Functional } \\
\text { recovery and } \\
\text { strengthening } \\
\text { exercises for } \\
\text { quadriceps and } \\
\text { hip muscles; } \\
\text { patellar } \\
\text { bandage; } \\
\text { patellofemoral, } \\
\text { tibiofemoral } \\
\text { JM (without } \\
\text { specifications) } \\
\text { and soft tissue; } \\
\text { education in } \\
\text { OA }\end{array}$ & $\begin{array}{l}\text { Education in } \\
\text { OA }\end{array}$ & $\begin{array}{l}\text { Global } \\
\text { perception; } \\
\text { Movement } \\
\text { pain } \\
\text { intensity; } \\
\text { Activities of } \\
\text { daily living; } \\
\text { Adverse } \\
\text { events and } \\
\text { use of drugs }\end{array}$ & $\begin{array}{l}\text { Likert scale; } \\
\text { VAS; } \\
\text { KOOS-ADL; } \\
\text { Physiotherapy } \\
\text { attendance, } \\
\text { home } \\
\text { exercises, } \\
\text { description } \\
\text { of adverse } \\
\text { events and } \\
\text { the medicines } \\
\text { used }\end{array}$ & $\begin{array}{l}\text { The EG reported a } \\
\text { higher percentage } \\
\text { of the item "greater } \\
\text { improvement" of } \\
\text { the general clinical } \\
\text { signs on the Likert } \\
\text { scale and greater } \\
\text { reduction of pain } \\
\text { when compared to } \\
\text { CG. There was no } \\
\text { significant effect } \\
\text { on ADLs. After } 9 \\
\text { months, there was no } \\
\text { significant effect on } \\
\text { self-reported pain }\end{array}$ & $\begin{array}{l}\text { After } 3 \text { months of } \\
\text { treatment, the EG } \\
\text { presented a superior } \\
\text { result in global } \\
\text { perception of clinical } \\
\text { change and pain } \\
\text { when compared to } \\
\text { CG. However, after } \\
6 \text { months, there was } \\
\text { no maintenance of } \\
\text { the effects observed } \\
\text { previously either in } \\
\text { physical function } \\
\text { and/or other positive } \\
\text { effects. }\end{array}$ \\
\hline $\begin{array}{l}\text { Farooq } \\
\text { et al. }{ }^{42}\end{array}$ & $\begin{array}{l}\text { Total }(n=68) \\
E G(n=34) \\
\text { and CG } \\
(n=34)\end{array}$ & $\begin{array}{l}\text { Chronic neck } \\
\text { pain }\end{array}$ & $\begin{array}{l}\text { Physiotherapy } \\
\text { (Infrared, } \\
\text { TUS, TENS, } \\
\text { isometric } \\
\text { exercises for } \\
\text { neck); } \\
\text { Cervical } \\
\text { mobilization } \\
\text { (Maitland } \\
\text { posteroanterior } \\
\text { oscillatory } \\
\text { mobilization), } \\
\text { participant } \\
\text { education, } \\
\text { home } \\
\text { exercises }\end{array}$ & $\begin{array}{l}\text { Physiotherapy } \\
\text { participant } \\
\text { education, } \\
\text { home } \\
\text { exercises }\end{array}$ & $\begin{array}{l}\text { Cervical pain } \\
\text { intensity at } \\
\text { rest; } \\
\text { Neck } \\
\text { disability } \\
\text { level; } \\
\text { Cervical } \\
\text { ROM; } \\
\text { cervical } \\
\text { muscular } \\
\text { endurance; } \\
\text { Analgesic } \\
\text { intake during } \\
\text { treatment }\end{array}$ & $\begin{array}{l}\text { VAS; } \\
\text { NDI; } \\
\text { Universal } \\
\text { goniometer; } \\
\text { Muscle } \\
\text { endurance } \\
\text { tests }\end{array}$ & $\begin{array}{l}\text { There was a } \\
\text { greater significant } \\
\text { reduction of pain } \\
\text { and disability in EG } \\
\text { when compared to } \\
\text { control group, as well } \\
\text { as an increase in the } \\
\text { resistance of cervical } \\
\text { muscles and cervical } \\
\text { ROM compared with } \\
\text { CG. All outcomes } \\
\text { had significant } \\
\text { improvement in both } \\
\text { groups. However, a } \\
\text { larger increase was } \\
\text { observed in the EG. }\end{array}$ & $\begin{array}{l}\text { The combination of } \\
\text { cervical mobilization } \\
\text { with physical therapy } \\
\text { is more effective } \\
\text { in reducing pain, } \\
\text { disability, muscular } \\
\text { resistance and ROM } \\
\text { in patients with } \\
\text { chronic mechanical } \\
\text { neck pain compared } \\
\text { to the group treated } \\
\text { only with } \\
\text { physiotherapy }\end{array}$ \\
\hline $\begin{array}{l}\text { Horst et } \\
\text { al. }{ }^{48}\end{array}$ & $\begin{array}{l}\text { Total }(n=72) \\
E G(n=36) \\
\text { and CG } \\
(n=36 ; 3 \\
\text { months } \\
\text { follow-up EG } \\
(n=33) \text { and } \\
\text { CG }(n=33)\end{array}$ & $\begin{array}{l}\text { Frozen } \\
\text { Shoulder }\end{array}$ & $\begin{array}{l}\text { Activity- } \\
\text { oriented } \\
\text { therapy } \\
\text { (strengthening } \\
\text { of shoulder } \\
\text { muscles, } \\
\text { several verbal } \\
\text { commands } \\
\text { for specific } \\
\text { movements of } \\
\text { the shoulder } \\
\text { and scapula), } \\
\text { aerobic } \\
\text { training, } \\
\text { cryotherapy, } \\
\text { laser therapy } \\
\text { and exercise } \\
\text { with elastic } \\
\text { bands }\end{array}$ & $\begin{array}{l}\text { Structural } \\
\text { oriented } \\
\text { therapy } \\
\text { (PNF, verbal } \\
\text { feedback, } \\
\text { passive } \\
\text { anteroposterior } \\
\text { humerus and } \\
\text { scapula JM, } \\
\text { separation } \\
\text { training and } \\
\text { joint approach), } \\
\text { aerobic } \\
\text { training, } \\
\text { cryotherapy, } \\
\text { laser therapy } \\
\text { and exercise } \\
\text { with elastic } \\
\text { bands }\end{array}$ & $\begin{array}{l}\text { Pain intensity } \\
\text { at rest; } \\
\text { Upper limbs } \\
\text { functionality; } \\
\text { ROM; } \\
\text { Muscle } \\
\text { strength }\end{array}$ & $\begin{array}{l}\text { McGill Pain } \\
\text { Questionnaire; } \\
\text { Upper } \\
\text { Extremity } \\
\text { Motor Activity } \\
\text { Log modified; } \\
\text { Goniometer; } \\
\text { Daniels and } \\
\text { Worthingham } \\
\text { muscle test }\end{array}$ & $\begin{array}{l}\text { The activity-oriented } \\
\text { group achieved } \\
\text { significant increases } \\
\text { in functional } \\
\text { performance and } \\
\text { activities of daily } \\
\text { living compared to } \\
\text { control group after } 10 \\
\text { days of therapy and } \\
\text { in the follow-up of } \\
\text { three months }\end{array}$ & $\begin{array}{l}\text { An activity-oriented } \\
\text { therapy program has } \\
\text { longer benefits than } \\
\text { targeted structural } \\
\text { therapy }\end{array}$ \\
\hline $\begin{array}{l}\text { Mayor et } \\
\text { al. }^{43}\end{array}$ & $\begin{array}{l}\text { Total }(n=90) \\
E G(n=45) \\
\text { and } C G(n= \\
42)\end{array}$ & $\begin{array}{l}\text { Mechanical } \\
\text { neck pain }\end{array}$ & $\begin{array}{l}\text { Manual } \\
\text { therapy } \\
\text { (cervical } \\
\text { mobilizations } \\
\text { (technique site } \\
\text { not specified), } \\
\text { neuromuscular } \\
\text { techniques, } \\
\text { stretches } \\
\text { and invasive } \\
\text { treatments } \\
\text { of trigger } \\
\text { points), home } \\
\text { exercises } \\
\text { and postural } \\
\text { guidelines }\end{array}$ & $\begin{array}{l}\text { TENS (F: } 80 \\
\text { Hz, T: } \leq 150 \\
\mu s, \text { adjustable } \\
\text { intensity), } \\
\text { home exercises } \\
\text { and postural } \\
\text { guidelines }\end{array}$ & $\begin{array}{l}\text { Pain intensity } \\
\text { at rest; } \\
\text { Neck } \\
\text { disability } \\
\text { level; } \\
\text { Quality of life; } \\
\text { Depression } \\
\text { and anxiety; } \\
\text { Drug use } \\
\text { (active } \\
\text { principle and } \\
\text { periodicity); } \\
\text { Expectation } \\
\text { of treatment; } \\
\text { Adverse } \\
\text { events }\end{array}$ & $\begin{array}{l}\text { VAS; } \\
\text { NDI; } \\
\text { SF-12; } \\
\text { GHQ-28; } \\
\text { Records } \\
\text { (drugs } \\
\text { prescribed by } \\
\text { physicians, } \\
\text { periodicity of } \\
\text { consumption, } \\
\text { adherence to } \\
\text { recommended } \\
\text { postural } \\
\text { care and } \\
\text { recommended } \\
\text { exercise) }\end{array}$ & $\begin{array}{l}\text { There was a } \\
\text { significant difference } \\
\text { in reduction of pain } \\
\text { intensity in both } \\
\text { groups }\end{array}$ & $\begin{array}{l}\text { Treatment with TENS } \\
\text { and manual therapy } \\
\text { produces a significant } \\
\text { reduction in pain } \\
\text { intensity, and there } \\
\text { are no differences } \\
\text { between these } \\
\text { treatment groups }\end{array}$ \\
\hline
\end{tabular}


Table 1. Characteristics of the included studies - continuation

\begin{tabular}{|c|c|c|c|c|c|c|c|c|}
\hline Authors & Sample & $\begin{array}{l}\text { Chronic pain } \\
\text { condition }\end{array}$ & $\begin{array}{l}\text { Interventions } \\
\text { description }\end{array}$ & $\begin{array}{l}\text { Control } \\
\text { Description }\end{array}$ & Outcomes & Instruments & Results & Conclusion \\
\hline $\begin{array}{l}\text { Richer, } \\
\text { Marchand and } \\
\text { Descarreaux }^{15}\end{array}$ & $\begin{array}{l}\text { Total }(n=37) \\
E G(n=19) \\
\text { and CG } \\
(n=18), 3 \\
\text { months } \\
\text { follow-up } \\
E G(n=15) \\
\text { and CG } \\
(n=12)\end{array}$ & $\begin{array}{l}\text { Chronic lateral } \\
\text { epicondylitis }\end{array}$ & $\begin{array}{l}\text { Anteroposterior } \\
\text { elbow } \\
\text { mobilization } \\
\text { (Mill } \\
\text { manipulation } \\
\text { described by } \\
\text { James Cyriax), } \\
\text { cryostimulation } \\
\text { with cryospray } \\
\text { at the trigger } \\
\text { point }\end{array}$ & $\begin{array}{l}\text { Ischemic } \\
\text { pressure } \\
\text { at the } \\
\text { myofascial } \\
\text { trigger } \\
\text { point }\end{array}$ & $\begin{array}{l}\text { Pain intensity } \\
\text { at rest; } \\
\text { gripping } \\
\text { force } \\
\text { without pain; } \\
\text { functional } \\
\text { outcomes } \\
\text { (disability } \\
\text { and pain) }\end{array}$ & $\begin{array}{l}\text { VAS; Hand } \\
\text { dynamometer; } \\
\text { PRTEE }\end{array}$ & $\begin{array}{l}\text { Significant } \\
\text { reduction of pain } \\
\text { and functional } \\
\text { index were } \\
\text { observed in } \\
\text { both groups } \\
\text { post intervention } \\
\text { evaluation and } \\
\text { were maintained } \\
\text { at follow-up. }\end{array}$ & $\begin{array}{l}\text { Based on preliminary } \\
\text { data from this study, } \\
\text { the combination } \\
\text { of cryostimulation } \\
\text { treatment and manual } \\
\text { therapy does not } \\
\text { provide short- and } \\
\text { long-term benefits. } \\
\text { The manual myofascial } \\
\text { point treatment and } \\
\text { mobilization techniques } \\
\text { provided positive } \\
\text { results in chronic lateral } \\
\text { epicondylitis. }\end{array}$ \\
\hline $\begin{array}{l}\text { Shashua et } \\
\text { al. }{ }^{40}\end{array}$ & $\begin{array}{l}\text { Total }(n=50) \\
E G(n=25) \\
\text { and CG } \\
(n=25) ; 6 \\
\text { months } \\
\text { follow-up } \\
\text { EG }(n=23) \\
\text { and CG } \\
(n=23)\end{array}$ & $\begin{array}{l}\text { Plantar } \\
\text { fasciitis }\end{array}$ & $\begin{array}{l}\text { TUS, } \\
\text { stretching and } \\
\text { anteroposterior } \\
\text { talocrural JM }\end{array}$ & $\begin{array}{l}\text { TUS and } \\
\text { stretching }\end{array}$ & $\begin{array}{l}\text { Pain intensity } \\
\text { at rest; } \\
\text { Dorsiflexion } \\
\text { ROM; } \\
\text { Lower limbs } \\
\text { functionality; } \\
\text { PPT }\end{array}$ & $\begin{array}{l}\text { NS; } \\
\text { Inclinometer; } \\
\text { LEFS; } \\
\text { Pressure } \\
\text { algometer }\end{array}$ & $\begin{array}{l}\text { No significant } \\
\text { differences were } \\
\text { found between } \\
\text { groups in any } \\
\text { outcome. Both } \\
\text { groups showed } \\
\text { a difference in } \\
\text { pain intensity } \\
\text { and lower limb } \\
\text { function. Both } \\
\text { groups increased } \\
\text { dorsiflexion } \\
\text { ROM, but there } \\
\text { was no difference } \\
\text { between groups }\end{array}$ & $\begin{array}{l}\text { The addition of ankle } \\
\text { and foot JM with } \\
\text { the aim of improving } \\
\text { dorsiflexion ROM is } \\
\text { no more effective than } \\
\text { the TUS treatment and } \\
\text { stretching only. The } \\
\text { association between } \\
\text { limitation of dorsiflexion } \\
\text { and plantar fasciitis } \\
\text { probably occurs } \\
\text { because of soft tissue } \\
\text { limitation and not from } \\
\text { the joint }\end{array}$ \\
\hline $\begin{array}{l}\text { Snodgrass et } \\
\text { al. }{ }^{41}\end{array}$ & $\begin{array}{l}\text { Total }(n=64) \\
\text { EG high } \\
\text { force } \\
\text { mobilization } \\
(n=21) \text {, } \\
\text { low force } \\
\text { mobilization } \\
(n=22), C G \\
(n=21) ; 4 \\
\text { days follow- } \\
\text { up EG: } \\
\text { high force } \\
\text { mobilization } \\
(n=20) \text { and } \\
\text { low force } \\
\text { mobilization } \\
(n=22), C G \\
(n=20)\end{array}$ & Neck pain & $\begin{array}{l}\text { Postero- } \\
\text { anterior JM } \\
\text { (grade III) in } \\
\text { cervical spine } \\
\mathrm{C} 7 \text { vertebra } \\
\text { with force of } 30 \\
\mathrm{~N} \text { or } 90 \mathrm{~N}\end{array}$ & $\begin{array}{l}\text { Laser } \\
\text { treatment } \\
\text { turned off }\end{array}$ & $\begin{array}{l}\text { PPT (spinous } \\
\text { process of } \\
\text { the cervical } \\
\text { vertebra, } \\
\text { upper } \\
\text { trapezius } \\
\text { muscle right, } \\
\text { median right } \\
\text { nerve trunk } \\
\text { in the elbow); } \\
\text { Pain intensity } \\
\text { at rest; } \\
\text { Cervical } \\
\text { ROM; } \\
\text { Cervical } \\
\text { stiffness; } \\
\text { Neck } \\
\text { disability } \\
\text { level }\end{array}$ & $\begin{array}{l}\text { Algometer; } \\
\text { VAS; } \\
\text { Cervical ROM } \\
\text { instrument; } \\
\text { Custom } \\
\text { device; } \\
\text { NDI }\end{array}$ & $\begin{array}{l}\text { At follow-up, the } \\
90 \mathrm{~N} \text { group had } \\
\text { lower pain than } \\
30 \mathrm{~N} \text { group and } \\
\text { lower cervical } \\
\text { stiffness than } \\
\text { the control } \\
\text { group. There was } \\
\text { no significant } \\
\text { difference } \\
\text { between the } \\
\text { groups in LDP } \\
\text { and WMD after } \\
\text { treatment or at } \\
\text { follow-up }\end{array}$ & $\begin{array}{l}\text { A specific dose of JM } \\
\text { in terms of applied } \\
\text { force seems necessary } \\
\text { to reduce stiffness } \\
\text { and potentially pain in } \\
\text { patients with chronic } \\
\text { neck pain. The changes } \\
\text { were not observed } \\
\text { immediately after the } \\
\text { mobilization, suggesting } \\
\text { that their effects are not } \\
\text { directly mechanical }\end{array}$ \\
\hline Sterling et al. ${ }^{49}$ & $\begin{array}{l}\text { Total }(n=34) \\
\text { EG }(n=19) \\
\text { and CG } \\
(n=15)\end{array}$ & $\begin{array}{l}\text { Chronic } \\
\text { whiplash } \\
\text { associated } \\
\text { disorders }\end{array}$ & $\begin{array}{l}\text { Cervical lateral } \\
\text { glide at the C5- } \\
\text { C6 level }\end{array}$ & $\begin{array}{l}\text { Manual } \\
\text { contact }\end{array}$ & $\begin{array}{l}\text { Pain and } \\
\text { disability } \\
\text { in cervical } \\
\text { spine; } \\
\text { Emotional } \\
\text { distress; } \\
\text { Pressure } \\
\text { pain } \\
\text { threshold; } \\
\text { Thermal pain } \\
\text { threshold; } \\
\text { NFR; pain } \\
\text { associated to } \\
\text { NFR test }\end{array}$ & $\begin{array}{l}\text { NDI; } \\
\text { GHQ-28; } \\
\text { Algometer; } \\
\text { Thermotest } \\
\text { system; } \\
\text { EMG; } \\
\text { VAS }\end{array}$ & & $\begin{array}{l}\text { Manual cervical lateral } \\
\text { glide technique has } \\
\text { the ability to modulate } \\
\text { spinal hyperexcitability } \\
\text { in patients with chronic } \\
\text { whiplash injury in short } \\
\text { term. However, manual } \\
\text { cervical lateral glide is } \\
\text { not recommended until } \\
\text { its long-term effects are } \\
\text { discovered and whether } \\
\text { they are equivalent } \\
\text { to reduced pain and } \\
\text { cervical inability }\end{array}$ \\
\hline Tavares et al. ${ }^{37}$ & $\begin{array}{l}\text { Total }(n=60) \\
E G(n=20), \\
\text { placebo } \\
\text { group } \\
(n=20), \text { and } \\
\text { CG }(n=20)\end{array}$ & $\begin{array}{l}\text { Chronic low } \\
\text { back pain }\end{array}$ & $\begin{array}{l}\text { Posteroanterior } \\
\text { central JM } \\
\text { grade II (for } 30 \\
\text { seconds on } \\
\text { each lumbar } \\
\text { vertebra L5 } \\
\text { to S1) }\end{array}$ & $\begin{array}{l}\text { Placebo: } \\
\text { reproduced } \\
\text { the same } \\
\text { positioning } \\
\text { of the } \\
\text { hands used } \\
\text { in the EG } \\
\text { without } \\
\text { rhythmic } \\
\text { oscillations } \\
\text { and with } \\
\text { the hands } \\
\text { at rest; } \\
\text { CG: without } \\
\text { intervention }\end{array}$ & $\begin{array}{l}\text { Pain intensity } \\
\text { at rest; } \\
\text { Low back } \\
\text { pain-related } \\
\text { incapacity; } \\
\text { Pain-related } \\
\text { catastrophi- } \\
\text { zing }\end{array}$ & $\begin{array}{l}\text { NS; } \\
\text { ODI; } \\
\text { PCS }\end{array}$ & & $\begin{array}{l}\text { JM was effective in } \\
\text { improving disability, } \\
\text { pain intensity and pre } \\
\text { and post-intervention } \\
\text { catastrophizing. In } \\
\text { comparison of the } \\
\text { effects between } \\
\text { intervention groups, } \\
\text { a reduction on pain } \\
\text { intensity was observed in } \\
\text { mobilization and placebo } \\
\text { groups in relation to CG, } \\
\text { suggesting a placebo } \\
\text { effect associated to } \\
\text { mobilization }\end{array}$ \\
\hline
\end{tabular}


Table 1. Characteristics of the included studies - continuation

\begin{tabular}{|c|c|c|c|c|c|c|c|c|}
\hline Authors & Sample & $\begin{array}{l}\text { Chronic pain } \\
\text { condition }\end{array}$ & $\begin{array}{l}\text { Interventions } \\
\text { description }\end{array}$ & $\begin{array}{l}\text { Control } \\
\text { Description }\end{array}$ & Outcomes & Instruments & Results & Conclusion \\
\hline $\begin{array}{l}\text { Villafañe } \\
\text { et al. }{ }^{45}\end{array}$ & $\begin{array}{l}\text { Total }(n=29) \\
\text { EG }(n=18) \text { and } \\
\text { CG }(n=18 ; 1 \\
\text { week follow-up } \\
\text { EG }(n=14) \text { and } \\
\text { CG }(n=15) ; 2 \\
\text { weeks follow- } \\
\text { up EG }(n=14) \\
\text { and } C G(n=15)\end{array}$ & $\begin{array}{l}\text { Secondary } \\
\text { thumb carpo- } \\
\text { metacarpal } \\
\text { OA }\end{array}$ & $\begin{array}{l}\text { Grade III } \\
\text { Kaltenborn JM } \\
\text { (anteroposte- } \\
\text { rior glide with } \\
\text { carpometa- } \\
\text { carpal joint } \\
\text { traction) }\end{array}$ & $\begin{array}{l}\text { TUS in non- } \\
\text { therapeutic } \\
\text { doses }\end{array}$ & $\begin{array}{l}\text { Pressure } \\
\text { pain thre- } \\
\text { shold; } \\
\text { Pinch and } \\
\text { grip force }\end{array}$ & $\begin{array}{l}\text { Algometer; } \\
\text { Pinch } \\
\text { dynamometer, } \\
\text { Hand } \\
\text { dynamometer }\end{array}$ & & $\begin{array}{l}\text { Kaltenborn JM reduced } \\
\text { pain in carpometacarpal } \\
\text { joint and the scaphoid } \\
\text { bone area. Thus, } \\
\text { mobilization can be } \\
\text { effective in reducing pain } \\
\text { and potentially improving } \\
\text { function in OA }\end{array}$ \\
\hline $\begin{array}{l}\text { Villafañe } \\
\text { et al. }{ }^{44}\end{array}$ & $\begin{array}{l}\text { Total }(n=28) \\
\text { EG }(n=14) \text { and } \\
\text { CG }(n=14) ; 1 \\
\text { week follow-up } \\
(E G(n=14) \text { and } \\
\text { CG }(n=14) ; 2 \\
\text { weeks follow- } \\
\text { up EG }(n=14) \\
\text { and CG }(n=14)\end{array}$ & $\begin{array}{l}\text { Thumb car- } \\
\text { pometacarpal } \\
\text { OA }\end{array}$ & $\begin{array}{l}\text { Maitland pos- } \\
\text { tero-anterior } \\
\text { trapeziometa- } \\
\text { carpal JM }\end{array}$ & $\begin{array}{l}\text { TUS in non- } \\
\text { therapeutic } \\
\text { doses }\end{array}$ & $\begin{array}{l}\text { PPT } \\
\text { Pinch and } \\
\text { grip stren- } \\
\text { gth. * in the } \\
\text { symptomatic } \\
\text { limb }\end{array}$ & $\begin{array}{l}\text { Algometer; } \\
\text { Pinch } \\
\text { dynamometer, } \\
\text { Hand } \\
\text { dynamometer }\end{array}$ & & $\begin{array}{l}\text { Accessory passive } \\
\text { mobilization increases } \\
\text { PPT at carpometacarpal } \\
\text { joint of thumb. However, } \\
\text { therapy does not } \\
\text { increase motor function } \\
\text { in patients with thumb } \\
\text { carpometacarpal OA }\end{array}$ \\
\hline $\begin{array}{l}\text { Villafañe } \\
\text { et al. }{ }^{47}\end{array}$ & $\begin{array}{l}\text { Total }(n=28) \\
\text { EG }(n=14) \text { and } \\
\text { CG }(n=14) ; 1 \\
\text { week follow-up } \\
\text { EG }(n=14) \text { and } \\
\text { CG }(n=14) ; 2 \\
\text { weeks follow- } \\
\text { up EG }(n=14) \\
\text { and CG }(n=14)\end{array}$ & $\begin{array}{l}\text { Thumb car- } \\
\text { pometacarpal } \\
\text { OA }\end{array}$ & $\begin{array}{l}\text { Carpometa- } \\
\text { carpal JM with } \\
\text { anteroposte- } \\
\text { rior slide }\end{array}$ & $\begin{array}{l}\text { Simulated } \\
\text { technique and } \\
\text { TUS in thumb } \\
\text { region }\end{array}$ & $\begin{array}{l}\text { PPT in } \\
\text { carpometa- } \\
\text { carpal joint, } \\
\text { in scaphoid } \\
\text { and hamato } \\
\text { bones; } \\
\text { Pinch and } \\
\text { grip force; } \\
\text { *asymptoma- } \\
\text { tic limb }\end{array}$ & $\begin{array}{l}\text { Algometer; } \\
\text { Pinch } \\
\text { dynamometer, } \\
\text { Hand } \\
\text { dynamometer }\end{array}$ & & $\begin{array}{l}\text { The application } \\
\text { of unilateral } \\
\text { accessory passive } \\
\text { mobilization directed } \\
\text { to the symptomatic } \\
\text { carpometacarpal joint } \\
\text { provided an increase } \\
\text { in PPT } 2 \text { weeks after } \\
\text { treatment; however, the } \\
\text { differences were small and } \\
\text { of limited clinical value. No } \\
\text { contralateral motor effects } \\
\text { were observed }\end{array}$ \\
\hline
\end{tabular}

SPADI = Shoulder Pain and Disability Index; NS = Numeric Scale; SF-36 = Medical Outcomes Study 36-item short form; AQoL = Assessment of Quality of Life; TUG test = Time Up and Go test; SPWT = Self Placed Walk test; OA = Osteoarthritis; TENS = Transcutaneous Electrical Nerve Stimulation; TUS = Therapeutic Ultrasound; $\mathrm{ROM}=$ Range of Motion; VAS = Visual Analog Scale; JM = Joint Mobilization; KOSS-ADL = Activities of Dailu Living subscale of the Knee Injury and Osteoarthritis Outcome Score; NDI = Neck Disability Index; PNF = Proprioceptive Neuromuscular Facilitation; SF-12 = Health Questionnaire SF-12; GHQ-28 = Goldberg Depression and Anxiety Scale; LEFS = Lower Extremity Functional Scale; PRTEE = Patient Rated Tennis Elbow Evaluation; PPT = Pressure Pain Threshold; EMG = Electromyography; CHQ-28 = General Health Questionnaire 28; PCS = Pain Catastrophizing Scale; ODI = Oswestry Disability Index; EG = experimental group; CG = control group; $A D L=$ activities of daily living; NFR = nociceptive flexion reflex.

\section{PRIMARY OUTCOMES}

\section{Pain intensity}

The majority of included trials (11 of 14) measured pain intensity only at rest ${ }^{15,36,38-45,48}$. One study measured the intensity of pain during movement (39), and one study evaluated pain intensity on movement and at rest ${ }^{36}$. Five studies evaluated the intensity of pain through the visual analog scale (VAS) ${ }^{15,39,41-43}$ and other four studies used the numerical rating scale (NRS) ${ }^{36,38-40}$. One study evaluated pain through the McGill Pain Questionnai$\mathrm{re}^{48}$. In study ${ }^{49}$ VAS was used to evaluate pain intensity associated with nociceptive flexion reflex test ${ }^{49}$.

All studies have shown significant short- and/or long-term reduction of pain intensity following JM combined or not with other therapies ${ }^{15,36,38-45,48}$.

\section{Pressure pain threshold (PPT)}

Some studies have evaluated PPT as a primary outco$\mathrm{me}^{40,41,44,46,47,49}$. This variable was measured through a digital pressure algometer at different points, according to pain location. The study ${ }^{49}$ evaluated PPT in patients with chronic cervical disorders related to whiplash injury. Three measurements were conducted in the C6 spinous process at the median nerve trunk bilaterally elbow and the tibialis anterior muscles bilaterally at two times before and after therapy ${ }^{49}$.
The study ${ }^{46}$ collected the measurements of PPT in the carpometacarpal joint at the bottom of the anatomical snuffbox and tubercle of the scaphoid bone. Three measurements were performed in the dominant hand of subjects with a one-minute interval, four times before treatment, 5 minutes after the treatment, 1 and 2 weeks after therapy.

On study ${ }^{44}$ the PPT was measured three times, with a rest interval of one minute between measurements, in bones connected to trapeziometacarpal joint, of scaphoid bone apophysis and hamato bone in four moments (baseline, immediately after treatment, one and two weeks after treatment) on symptomatic hand with OA carpometacarpal.

Another study ${ }^{47}$ consisted of a secondary analysis that focused on the contralateral hand (asymptomatic). The PPT was evaluated three times with a rest interval of one minute between measurements on the carpometacarpal joint of the contralateral thumb and the symptomatic hand with carpometacarpal OA, on scaphoid bone tubercle and hamato bone process in four moments (baseline, immediately after treatment, one and two weeks after treatment).

On study ${ }^{41}$ PPT measurements were performed three times, with an interval of 10 seconds between measurements in the following points: close to spinous process of cervical spine at medullary level treated on right side, with participant in pronated position, upper right trapezius muscle, between $\mathrm{C} 7$ and acromion, with sitting participant and trunk of the right median nerve at the 
elbow, medial to the biceps tendon, with the elbow at approximately $30^{\circ}$ flexion, with forearm resting on a support and participant sitting in three moments (before, after and follow-up). The study ${ }^{40}$ performed three PPT measurements with a 30 -second interval between each application at the pain site in patients with plantar fasciitis. Algometry was measured twice (baseline and at the end of all care sessions).

Five of six studies ${ }^{40,41,44.46}$ demonstrated a significant increase in short and/or long-term pressure pain threshold after JM application, associated or not with other therapies. Only one study ${ }^{49}$ showed no significant difference after the use of this therapy.

\section{SECONDARY OUTCOMES}

\section{Range of motion}

Five of the included studies performed ROM measurement $^{38,40-42,48}$. Among studies that evaluated ROM with a universal goniometer, one article evaluated only active mobility ${ }^{42}$, and two other studies did not specified ${ }^{38,48}$. Active ROM was still evaluated through the inclinometer in a study ${ }^{40}$ and through the cervical range of motion instrument ${ }^{41}$.

Four articles showed a significant increase of ROM at cervical ${ }^{142}$, shoulder ${ }^{48}$, hip ${ }^{38}$, and foot ${ }^{40}$ joints after JM application associated or not with other therapies. One study ${ }^{41}$ did not find any significant difference for cervical ROM after therapy.

\section{Muscle strength}

Five studies evaluated muscle strength ${ }^{15,36,44,46-48}$. In the study ${ }^{36}$, the isometric strength of the symptomatic shoulder was assessed for abduction, internal and external rotation through the Nicholas Manual Muscle Tester (Lafayette, EUA) performed with a dynamometer. After the demonstration and training test, participants were asked to push as much as possible against the dynamometer for 4 seconds while the evaluator provided a verbal stimulus ${ }^{36}$.

The study ${ }^{48}$ used muscle testing procedures by Daniels and Worthingham to assess the strength of the major shoulder muscles. In this system, muscle strength is marked with a numerical rating ranging from zero, indicating no muscle activation, to 5 for the best possible response to manual resistance in a reduced range of the muscle group that performs the movement.

Other studies ${ }^{15,44,46,47}$ used the hand dynamometer to measure the strength of patients with chronic lateral epicondylitis and thumb metacarpal OA, respectively ${ }^{15,44,46,47}$. The studies from ${ }^{44,46,47}$ also used the tweezer dynamometer to evaluate thumb strength ${ }^{44,46,47}$. Increased muscle strength after JM application associated or not to other therapies was observed in three studies ${ }^{36,46,48}$. Three other studies found no changes in strength after therapy ${ }^{15,44,47}$.

\section{Functionality}

Ten studies that were included investigated hip, shoulder, knee, and foot functionality. Nine of them used questionnaires $^{36,37,39-43,48,49}$ and one used functional tests ${ }^{38}$, all with validation. The study ${ }^{38}$ on the immediate effects of mobilization in patients with hip OA was the only that evaluated the functional indexes of patients through validated test $s^{38}$. The Timed Up and Go test (TUG), which simulates some functional activities of daily living (from sitting to standing, walking and standing to sitting) ${ }^{38}$; the 30s Chair Stand Test (CST), which assesses function and strength of the lower limbs ${ }^{38}$ and the $40 \mathrm{~m}$ Self Placed Walk Test (SPWT), which measures the time required to walk on short distances ${ }^{38}$.

The other studies used the following questionnaires: Shoulder Pain and Disability Index (SPADI) to evaluate shoulder function in patients with chronic rotator cuff lesions ${ }^{36}$; Knee Injury and Osteoarthritis Outcome Score (KOOS) to evaluate knee function/performance in activities of daily living (ADL) in patients with patellofemoral $\mathrm{OA}^{39}$; the Neck Disability Index (NDI) was used to assess pain and disability of cervical spine in patients with chronic neck pain, cervicalgia and chronic whiplash injury disorders, respectively ${ }^{41-43,49}$; the Upper Extremity Motor Activity Log Modified to measure upper limb functionality in patients with frozen shoulder ${ }^{48}$; the Lower Extremity Functional Scale (LEFS) for assessing the functionality of lower limbs of patients with plantar fasciitis ${ }^{40}$; the Oswestry Disability Index (ODI) that was used in patients with chronic low back pain to measure pain-related disability in the lumbar spine ${ }^{37}$ and the Patient Rated Tennis Elbow Evaluation (PRTEE), which evaluated the functional outcomes related to pain and disability in patients with chronic lateral epicondylitis ${ }^{15}$.

Improvement of functionality was observed in seven stu$\operatorname{dies}^{36-38,40-42,48}$ after JM application associated or not with other therapies. There was no significant difference in functionality in three studies ${ }^{39,43,49}$ after JM application.

\section{Quality of life}

Three studies assessed quality of life (AQoL) $)^{36,39,43}$, using different instruments, such as the Medical Outcomes Study 36-item shor$\mathrm{t}$-form (SF-36) ${ }^{36}$, the Assessment of Quality of Life (AQoL) ${ }^{36}$, the ADL subscale of Knee injury and Osteoarthritis Outcome Score $(\text { KOOS })^{39}$ and the 12-item health survey $(\mathrm{SF}-12)^{43}$.

Only one study ${ }^{36}$ showed a significant increase in the quality of life parameters after JM application. Two studies ${ }^{39-43}$ did not show any significant difference in this outcome after therapy.

\section{Adherence to treatment}

Only two studies evaluated adherence of participants through medical records, considering the number of visits performed and the total number of visits that were pre-established in the study protocol $^{36,39}$. Both studies showed good patient adherence to JM treatment, but no significant difference was observed between treatment and control groups.

\section{Expectation/satisfaction}

Three studies evaluated the expectations before the intervention, relating to their respective satisfactions after intervention ${ }^{36,39,43}$ A five-point Likert scale was used $(1=$ much worse, $2=$ slightly worse, $3=$ no change, $4=$ slightly better, $5=$ much better $)^{36,39}$, this data was registered in each patient's medical records. The study ${ }^{43}$ evaluated the expectation before treatment from the concepts chosen by the patient: complete recovery, great improvement, partial relief or no expectation of relief. All three studies reported that most participants reported satisfaction and improvement with JM treatment performed. 


\section{Adverse events}

Only three studies reported adverse events ${ }^{36,39,43}$. In the stu$\mathrm{dy}^{43}, 16.3 \%$ of patients treated with transcutaneous electrical nerve stimulation (TENS) $(n=7)$ and $6.4 \%$ of those treated with manual therapy $(n=3)$ reported treatment-related adverse effects. Three of them presented increased pain in the treated area and one showed the general physical condition of the group treated with TENS. Of those who received manual therapy, one patient reported clinical worsening during the first few days and the others did not specify symptoms ${ }^{43}$.

Study ${ }^{36}$ reported that during the intervention period, 17 participants out of $55(31 \%)$ from the active group had adverse effects that included increased short-term pain during or after treatment $(n=3)$, increase in short-term pain with home workouts $(n=12)$ and slight irritation to tape used for postural taping $(n=2)$. In the placebo group, five participants out of $61(8 \%)$ reported adverse events involving increased short-term pain during or after treatment. During the follow-up period, 7 of 49 patients (14\%) from the active group reported adverse events and included increased short-term pain with home exercise $\mathrm{e}^{36}$.

In the study ${ }^{39}$ adverse events were observed in seven participants who undergone exercise, education, manual therapy, taping intervention (skin reaction to the use of tape $(n=2)$ ); edema after treatment $(n=2)$; pain in other areas after exercise (lumbar $n=1$; ankle $n=1$; another knee $n=1$ ). All adverse events were mild, with no need for medical intervention or treatment discontinuation (some bandage adjustments were performed and/or exercises were done by the physiotherapist).

Thus, most of the adverse effects caused slight damages to the patients in included studies, were generally related to the increase of local pain immediately after the technique application, but without lasting for a long time.

\section{Other variables}

All studies recorded demographic data (sex, age, body mass index, height, among others). Other variables were also considered in some studies, such as thermal pain threshold ${ }^{49}$, nociceptive flexion reflex and pain related to this test ${ }^{49}$, pain catastrophizing ${ }^{37}$, level of anxiety, and depression ${ }^{43,49}$ and use of drugs during treatment ${ }^{39,42,43}$. Two studies evaluated the levels of depression and anxiety in the participants through the Goldberg Depression and Anxiety Scale (GHQ-28). In one study, it was evidenced that $42.6 \%$ of the participants treated with manual therapy presented anxiety and depression ${ }^{43}$, and the other study showed that all individuals had high levels of anxiety and depression ${ }^{49}$.

The study ${ }^{49}$ evaluated the thermal threshold, nociceptive flexion reflex and the pain associated with this test and showed that there was an increase in the nociceptive reflex flexion threshold in the group treated with JM and that there was no significant difference in pain during the reflex test nociceptive flexion and at the threshold of thermal pain. On sutdy ${ }^{37}$ catastrophizing evaluation of patients with chronic low back pain was performed and pain catastrophizing interference was observed in the treatment of these patients

Three studies reported the use of drugs during treatment ${ }^{39,42,43}$. The study ${ }^{39}$ evidenced similar use of analgesics, non-steroidal anti-inflammatory drugs (NSAIDs), glucosamines, and fish oil.
The study ${ }^{42}$ use of pain medication was registered in five patients of both groups, and there was no significant difference between them. The study ${ }^{43}$ details the periodicity of the consumption of NSAIDs, analgesics and muscle relaxants. In the group treated with manual therapy, $4.3 \%$ of patients took these drugs every day, while $12.8 \%$ reported weekly or monthly consumption.

\section{Risk of bias}

The studies were evaluated for the risk of bias (low, high, or unclear) in relation to six domains. Figure 2 summarizes the results of individual studies.

One study adequately described all domains and was considered as a low risk of bias ${ }^{38}$. One study presented a high risk for the selection bias by using an open randomization process (random list of numbers) ${ }^{49}$. Two studies presented an unclear risk for the performance bias because they did not present information about

Figure 2. Risk of bias

\begin{tabular}{|c|c|c|c|c|c|c|c|}
\hline & 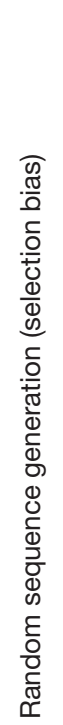 & 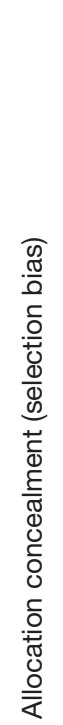 & 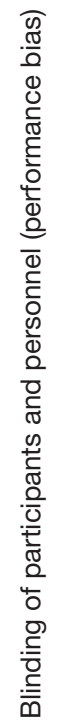 & 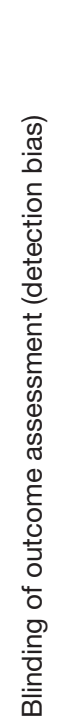 & 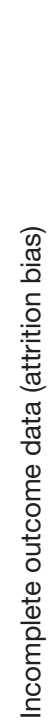 & 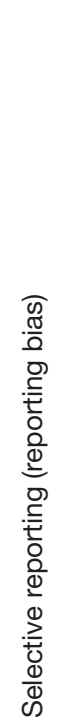 & 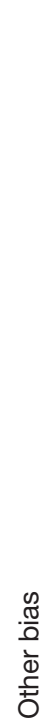 \\
\hline Bennell et al. ${ }^{36}$ & ( & ( & ( & $\oplus$ & $\oplus$ & † & $\odot$ \\
\hline Beselga et al. ${ }^{38}$ & ( & † & ( & $\oplus$ & $\oplus$ & ( & $\oplus$ \\
\hline Crossley et al. ${ }^{39}$ & † & $\oplus$ & $\oplus$ & ( & $\oplus$ & ( & $\odot$ \\
\hline Farooq et al. ${ }^{42}$ & $\oplus$ & $\oplus$ & $\oplus$ & $\oplus$ & $\oplus$ & ( & $\odot$ \\
\hline Horst et al. ${ }^{48}$ & † & ( & † & † & $\oplus$ & $\oplus$ & $\odot$ \\
\hline Mayor et al. ${ }^{43}$ & (†) & ( & ( & $\oplus$ & ( & $\oplus$ & $\odot$ \\
\hline $\begin{array}{l}\text { Richer, Marchand and } \\
\text { Descarreaux }{ }^{15}\end{array}$ & $\oplus$ & $\oplus$ & $\oplus$ & ( & $\oplus$ & $\oplus$ & $\odot$ \\
\hline Shashua et al. ${ }^{40}$ & † & ( & (?) & † & $\oplus$ & ( & $\odot$ \\
\hline Snodgrass et al. ${ }^{41}$ & ( & ( & $\oplus$ & $\oplus$ & $\oplus$ & ( & $\odot$ \\
\hline Sterling et al. ${ }^{49}$ & ( & $\Theta$ & ?? & ( & $\oplus$ & $\oplus$ & $\odot$ \\
\hline Tavares et al. ${ }^{37}$ & ( & † & ( & ( & $\oplus$ & ( & \\
\hline Villafane et al. ${ }^{45}$ & $\oplus$ & ( & $\oplus$ & ( & $\oplus$ & ( & $\odot$ \\
\hline Villafane et al. ${ }^{44}$ & † & ( & † & † & $\oplus$ & ( & $\odot$ \\
\hline Villafane et al. ${ }^{45}$ & ( & ( & $\oplus$ & ( & $\oplus$ & † & $\odot$ \\
\hline
\end{tabular}




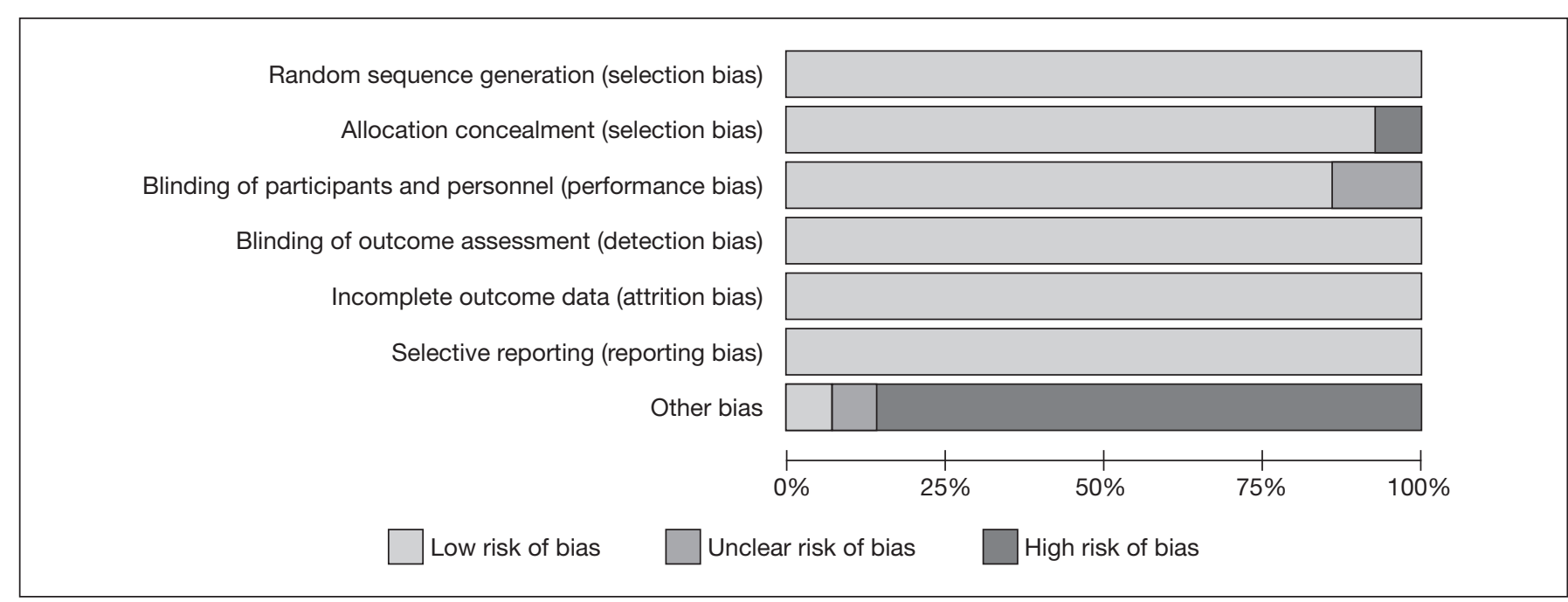

Figure 3. Risk of bias graph: review authors' judgments about each risk of bias item presented as percentages across all included studies

the blindness of participants and researchers ${ }^{40,49}$. All studies had a low risk of reporting bias ${ }^{15,36-44,46-49}$.

In the domain "other bias," 12 studies presented a high risk of bias $^{15,36,39-46,48,49}$. Six reported that more than one therapist performed the mobilization ${ }^{15,36,39,40,43,48}$, seven performed different types and techniques of JM without specifying the type of mobilization and/or combined with other therapies ${ }^{15,36,39,40,42,43,48}$, and 11 articles used different treatment controls, such as TENS, therapeutic ultrasound, laser, stretching or patient educa$\operatorname{tion}^{15,36,39-44,47-49}$. Three studies did not report the experience of the therapists who performed treatment ${ }^{37,46,49}$. One study was considered to be at an unclear risk because it did not report the number of therapists who performed the mobilization techniques and the time of clinical experience in the area ${ }^{37}$.

In general, the methodological quality of studies was considered a low risk of bias. The values referring to the percentage of articles classified in each degree of risk of bias are represented in figure 3 .

\section{DISCUSSION}

According to the studies analyzed, JM seems to have better results in the management of chronic musculoskeletal conditions when it was used alone or in combination with conventional physiotherapy. From the 14 articles included, seven used JM interventions alone compared to a control group using simulated placebo therapy ${ }^{37,38}$, other interventions with appliances switched off or in non-therapeutic doses ${ }^{41,44,46,47}$, manual contact ${ }^{49}$ or without treatment ${ }^{37}$ and seven used JM in association with therapeutic resources used in conventional physiotherapy (TENS, TUS, strengthening, patient education and home exercises) compared to the same therapy without $\mathrm{JM}^{15,36,39,40,42,43,48}$. This shows the scarcity of clinical trials that use only JM as treatment, which makes it difficult to know the efficacy of this technique alone in various types of musculoskeletal disorders. In addition, the lack of validated placebo techniques for JM also difficult the discovery of new evidence about technique effect.
For primary outcomes (pain intensity and pressure pain threshold), JM promoted short-term ${ }^{37,38,44,46,47}$ and long-term ${ }^{41}$ pain reduction in different types of musculoskeletal pain when only mobilization was used as treatment. When applied in combination with other therapies in musculoskeletal lower limb dysfunctions (patellofemoral OA and plantar fasciitis) and spine (chronic neck pain chronic disorders related to whiplash injury and chronic low back pain), the studies showed a reduction of pain after treatment $^{37,39,40,42,43,49}$. In upper limb dysfunctions (rotator cuff injury, frozen shoulder, lateral epicondylitis and thumb carpometacarpal OA), some studies have reported pain reduction ${ }^{15,46}$, and others did not observe any differences between groups ${ }^{36,42,43,48}$. Most of the articles also showed improvement of ROM, strength and function after JM treatment $t^{15,36-38,40-42,46}$. Based on these studies, most of them with good methodological quality, JM can be considered an effective therapeutic resource in reducing pain and improving the functionality of patients with CMP. There is insufficient evidence to determine the effect of JM on quality of life, adherence and patient expectancy in the treatment of CMP.

Previous reviews and meta-analyses show chronic musculoskeletal conditions studied separately and involving manual therapy as a set or combination of manual techniques (neck pain ${ }^{35,50}$, lower back pain ${ }^{14,51}$, patellofemoral pain ${ }^{52}$, impingement syndro$\mathrm{me}^{53}$, hip $\mathrm{OA}^{1}$, or using a particular technique of JM for pain treatment ${ }^{54}$. In addition, it presents restrictions on language and publication period of selected articles, and the search was performed in a small number of databases, which makes these studies limited. Differently, the present review performed the search on 12 databases, without year and publication language restrictions, which reduces the risk of loss of some article at screening and, consequently, provides greater access to the data extracted from studies already published about this theme.

The prevalence of common musculoskeletal conditions has a strong relationship with age, being directly proportional to aging, with an increase of painful complaints due to the wear of the musculoskeletal system ${ }^{3}$. In this review, neck pain was more prevalent among included studies ${ }^{41-43,40}$, in contrast to the number of back 
pain studies in the literature, who had only one study included ${ }^{37}$. This may have been caused by not meeting the inclusion criteria in this review or the fact that manipulation seems to be the most appropriate technique for low back pain according to current revisions ${ }^{14}$. Later appear the injuries that affect the shoulders, such as rotator cuff disease ${ }^{36}$ and frozen shoulder ${ }^{48}$ and to a less extent, diseases such as lateral epicondylagia ${ }^{15}$ and plantar fasciitis ${ }^{40}$. OA is a common condition shown in studies in different body regions and patellofemoral ${ }^{17}$, carpometacarpal $^{44-46}$ and hip ${ }^{16}$.

Autors $^{51}$ in a systematic review (SR) about JM and exercise efficacy for different stages of non-specific low back pain, found that JM (being the high speed and low amplitude manipulation of the pelvic loin region that presents evidence of moderate support compared to mobilization and soft tissue techniques including "myofascial," "miotensive" or "harmonic" techniques in this same body region), in combination with specific and/or general exercises, or usual medical care, are better than any of these isolated interventions.

In agreement, study ${ }^{14}$ in a meta-analysis on manipulation and mobilization in the treatment of chronic low back pain, have observed that the manipulation of high speed and low amplitude (thrust) is the most recommended. However, the search conducted in this SR showed year restriction (January 2000 to April 2013), language (only articles in English), and a few electronic databases (Medline, Cochrane Register of Controlled Trials, PEDro, CINAHL, PsycINFO, and ICL). In this meta-analysis, a specific population was not defined since there was no homogeneity in the causes of low back pain, which implies a greater heterogeneity of studies and consequently a higher methodological bias and less applicability of these data in clinical practice. In an SR about the use of exercise in mechanical neck pain, stu$\mathrm{dy}^{55}$ concluded that there was no evidence of high quality, indicating that there is still uncertainty about the efficacy of specific strengthening and resistance exercises for neck pain. Study ${ }^{50}$, in SR about JM efficacy and exercise for nonspecific cervical pain, emphasized the importance of performing combined treatment (JM plus exercises), being better compared to JM or exercises alone.

The authors also showed that JM does not need to be applied at the symptomatic level to improve pain and can then be applied at adjacent levels according to the irritability status ${ }^{50}$. These reviews also show that manipulation is less indicated in cervical disorders, recommending that the thoracic manipulation or mobilization should be performed. This agrees with results found in studies included in this $\mathrm{SR}^{41-43,49}$, who considered mobilization as a treatment with good results in patients with nonspecific mechanical neck pain and with whiplash injury-related cervical disorders, applied either alone or in association with conventional therapy.

In studies about chronic pain, pain intensity is considered the primary outcome in most clinical trials ${ }^{56}$ and should be investigated both at rest and during movement. From the included studies, 12 evaluated only pain at rest ${ }^{15,37,38,40-46,49}$, one study assessed only pain in motion ${ }^{39}$, and only one has evaluated pain at rest and during movement ${ }^{36}$. Both the intensity of pain in rest and during movement must be evaluated because there are pain conditions that do not occur or have lower intensity at rest, which may mask the evaluation and generate a bias in the study.
Therefore, it is very important to perform pain assessment in these two conditions in order to have more reliable data about the pain of a population.

In the reviews cited above, positive results were also observed for the reduction of pain associated with the use of manual therapy/ mobilization and combinations with exercises ${ }^{50}$. In a previous SR about the use of physiotherapeutic treatment in subacromial pain, it has been shown that exercise therapy should be the best treatment for pain reduction and improvement of function and range of motion. However, the addition of joint mobilizations to exercise can accelerate the reduction of short-term pain ${ }^{53}$. The same can be observed in studies included in this SR that combine the use of JM with conventional physiotherapy ${ }^{36,39,30,43,43,48}$. People with chronic pain present reduced functionality, and this loss can be evaluated through functional index instruments ${ }^{18}$. Of the 14 included studies, only seven assessed functionality ${ }^{36-40,48,49}$, which shows a deficiency in studies regarding the evaluation of this variable. This is evidenced by the Initiative on Methods, Measurement and Evaluation of Pain in Clinical Trials (IMMPACT) and Outcome Measures in Rheumatology (OMERACT), which bring the importance of assessing both pain and functionality of patients ${ }^{17}$.

A systematic review investigated the risks of manual treatment on the vertebral segment and concluded that serious adverse events are rare, the most common are mild, and these are associated with a greater amount of spinal manipulation ${ }^{57}$. Although some studies included in this $\mathrm{SR}^{36,39,43}$ have investigated adverse events as a secondary outcome and have reported as minimum, there are not enough data to prove this variable due to heterogeneity of the studies.

This review presented some strength points, including the development of the question and the population table, intervention, control/comparison and result (PICO strategy), use of a systematic, explicit and transparent methodology, incorporating internal validity evaluation (risk of bias), independent methodological evaluation by a third reviewer for each of the technical steps involved in the review phases, and a comprehensive survey in most databases, without restrictions. None of the authors reported any conflict of interest.

Nevertheless, there are some limitations in this systematic review that make it impossible to conduct a meta-analysis. Although most of the included studies present low risk of bias, there was a significant heterogeneity regarding the protocol of JM application (different degrees of mobilization, series, repetitions, body segments, type of mobilization - Mulligan, Maitland, Kaltenborn and passive), duration of the chronic condition, treatment performed by more than one professional with different experiences and training times, compared to control groups or placebo using other techniques or treatments that are not related to manual therapy and outcomes assessed in different ways.

From that, future clinical trials should aim to use only the JM technique in experimental groups to standardize the application protocols of the techniques and validate placebo techniques with the use of manual therapy. Based on that, studies can be performed with greater methodological accuracy regarding the application of the technique and can generate greater and more reliable results. 


\section{CONCLUSION}

According to the results of this review, JM seems to be an effective technique for CMP, when applied alone or in association with other interventions, once it causes pain intensity decrease, improvement on range of motion, strength, functionality, quality of life, with good patient adherence/satisfaction and low adverse events. Based on this review, no specific clinical recommendations can be made on the optimal dose of treatment through JM. Future clinical trials should investigate mobilization types and the dose of treatment according to different musculoskeletal diseases.

\section{Funding sources}

This research did not receive any specific grant from funding agencies in the public, commercial, or not-for-profit sectors.

\section{REFERENCES}

1. Sampath KK, Mani R, Miyamori T, Tumilty S. The effects of manual therapy or exercise therapy or both in people with hip osteoarthritis: a systematic review and meta-analysis. Clin Rehabil. 2016;30(12):1141-55.

2. Sahrmann S, Azevedo DC, Dillen LV. Diagnosis and treatment of movement system impairment syndromes. Braz J Phys Therapy. 2017;21(6):391-9.

3. Blyth FM, Noguchi N. Chronic musculoskeletal pain and its impact on older people. Best Pract Res Clin Rheumatol. 2017;31(2):160-8.

4. Cimmino MA, Ferrone C, Cutolo M. Epidemiology of chronic musculoskeletal pain. Best Pract Res Clin Rheumatol. 2011;25(2):173-83.

5. Toye F, Seers K, Allcock N, Briggs M, Carr E, Andrews J, et al. Patients' experiences of chronic non-malignant musculoskeletal pain: a qualitative systematic review. Br J Gen Pract. 2013;63(617):e829-e41.

6. Gerdle B, Ghafouri B, Ernberg M, Larsson B. Chronic musculoskeletal pain: review of mechanisms and biochemical biomarkers as assessed by the microdialysis technique. J Pain Res. 2014;7:313.

7. Crofford LJ. Chronic pain: where the body meets the brain. Trans Am Clin Climatol Assoc. 2015;126:167.

8. Clauw DJ. Diagnosing and treating chronic musculoskeletal pain based on the underlying mechanism(s). Best Pract Res Clin Rheumatol. 2015;29(1):6-19.

9. Hirase T, Kataoka H, Inokuchi S, Nakano J, Sakamoto J, Okita M. Factors associated with chronic musculoskeletal pain in Japanese community-dwelling older adults: A cross-sectional study. Medicine. 2017;96(23):e7069.

10. Geneen LJ, Moore RA, Clarke C, Martin D, Colvin LA, Smith BH. Physical activity and exercise for chronic pain in adults: an overview of Cochrane Reviews. Cochrane Database Syst Rev. 2017;4:CD011279.

11. Ushida T. Burdensome problems of chronic musculoskeletal pain and future prospects. J Orthop Sci. 2015;20(6):958-66.

12. Rajapakse D, Liossi C, Howard RF. Presentation and management of chronic pain. Arch Dis Child. 2014;99(5):474-80.

13. Krekoukias G, Gelalis ID, Xenakis T, Gioftsos G, Dimitriadis Z, Sakellari V. Spinal mobilization vs conventional physiotherapy in the management of chronic low back pain due to spinal disk degeneration: a randomized controlled trial. J Man Manip Ther. 2017;25(2):66-73

14. Coulter ID, Crawford C, Hurwitz EL, Vernon H, Khorsan R, Booth MS, et al. Manipulation and mobilization for treating chronic low back pain: a systematic review and meta-analysis. Spine J. 2018;18(5):866-79.

15. Richer N, Marchand AA, Descarreaux M. Management of chronic lateral epicondylitis with manual therapy and local cryostimulation: a pilot study. J Chiropr Med. 2017;16(4):279-88.

16. Wyke B, Polacek P. Articular neurology: the present position. J Joint Bone Surg. 1975;57(3):401

17. Taylor AM, Phillips K, Patel KV, Turk DC, Dworkin RH, Beaton D, et al. Assessment of physical function and participation in chronic pain clinical trials: IMMPACT/ OMERACT recommendations. Pain. 2016;157(9):1836-50.

18. Seroussi R. Chronic pain assessment. Phys Med Rehabil Clin N Am. 2015;26(2):185-99.

19. Kingston L, Claydon L, Tumilty S. The effects of spinal mobilizations on the sympathetic nervous system: a systematic review. Man Ther. 2014;19(4):281-7.

20. Lovick T. Interactions between descending pathways from the dorsal and ventrolateral periaqueductal gray matter in the rat. The midbrain periaqueductal gray matter: Springer; 1991. 101-20p.

21. Sterling M, Jull G, Wright A. Cervical mobilisation: concurrent effects on pain, sympathetic nervous system activity and motor activity. Man Ther. 2001;6(2):72-81.

22. Paungmali A, O’Leary S, Souvlis T, Vicenzino B. Hypoalgesic and sympathoexcitatory effects of mobilization with movement for lateral epicondylalgia. Phys Ther.
2003;83(4):374-83.

23. Slater H, Vicenzino B, Wright A. 'Sympathetic Slump': the effects of a novel manual therapy technique on peripheral sympathetic nervous system function. J Man Manip Ther. 1994;2(4):156-62.

24. Perry J, Green A, Singh S, Watson P. A randomised, independent groups study investigating the sympathetic nervous system responses to two manual therapy treatments in patients with LBP. Man Ther. 2015;20(6):861-7.

25. Tsirakis V, Perry J. The effects of a modified spinal mobilisation with leg movement (SMWLM) technique on sympathetic outflow to the lower limbs. Man Ther. 2015;20(1):103-8.

26. Piekarz V, Perry J. An investigation into the effects of applying a lumbar Maitland mobilisation at different frequencies on sympathetic nervous system activity levels in the lower limb. Man Ther. 2016;23(1):83-9.

27. Moutzouri M, Perry J, Billis E. Investigation of the effects of a centrally applied lumbar sustained natural apophyseal glide mobilization on lower limb sympathetic nervous system activity in asymptomatic subjects. J Manipulative Physiol Ther. 2012;35(4):286-94

28. Cleland J, Durall C, Scott SA. Effects of slump long sitting on peripheral sudomoto and vasomotor function: a pilot study. J Man Manip Ther. 2002;10(2):67-75.

29. Navarro-Santana MJ, Gómez-Chiguano GF, Somkereki MD, Fernández-de-las-Peńas C, Cleland JA, Plaza-Manzano G. Effects of joint mobilisation on clinical manifestations of sympathetic nervous system activity: a systematic review and meta-analysis. Physiotherapy. 2019. Epub ahead print)

30. Vicenzino B, Cartwright T, Collins D, Wright A. Cardiovascular and respiratory changes produced by lateral glide mobilization of the cervical spine. Man Ther. 1998;3(2):67-71.

31. La Touche R, París-Alemany A, Mannheimer JS, Angulo-Díaz-Parreño S, Bishop MD, Lopéz-Valverde-Centeno A, et al. Does mobilization of the upper cervical spine affect pain sensitivity and autonomic nervous system function in patients with cervico-craniofacial pain?: A randomized-controlled trial. Clin J Pain. 2013;29(3):205-15.

32. Roy RA, Boucher JP, Comtois AS. Paraspinal cutaneous temperature modification after spinal manipulation at L5. J Manipulative Physiol Ther. 2010;33(4):308-14.

33. Skyba D, Radhakrishnan R, Rohlwing J, Wright A, Sluka K. Joint manipulation reduces hyperalgesia by activation of monoamine receptors but not opioid or GABA receptors in the spinal cord. Pain. 2003;106(1-2):159-68.

34. Martins DF, Mazzardo-Martins L, Cidral-Filho FJ, Stramosk J, Santos AR. Ankle joint mobilization affects postoperative pain through peripheral and central adenosine A1 receptors. Phys Ther. 2013;93(3):401-12.

35. Fredin K, Lorås $\mathrm{H}$. Manual therapy, exercise therapy or combined treatment in the management of adult neck pain - a systematic review and meta-analysis. Musculoskelet Sci Pract. 2017;31:62-71.

36. Bennell K, Wee E, Coburn S, Green S, Harris A, Staples M, et al. Efficacy of standardised manual therapy and home exercise programme for chronic rotator cuff disease: randomised placebo controlled trial. BMJ. 2010;340:c2756

37. Tavares FA, Chaves TC, Silva ED, Guerreiro GD, Gonçalves JF, Albuquerque AA. Immediate effects of joint mobilization compared to sham and control intervention for pain intensity and disability in chronic low back pain patients: randomized controlled clinical trial. Rev Dor. 2017;18(1):2-7.

38. Beselga C, Neto F, Alburquerque-Sendín F, Hall T, Oliveira-Campelo N. Immediate effects of hip mobilization with movement in patients with hip osteoarthritis: a randomised controlled trial. Man Ther. 2016;22:80-5.

39. Crossley KM, Vicenzino B, Lentzos J, Schache AG, Pandy M, Ozturk H, et al Exercise, education, manual-therapy and taping compared to education for patellofemoral osteoarthritis: a blinded, randomised clinical trial. Osteoarthritis Cartilage. 2015;23(9):1457-64.

40. Shashua A, Flechter S, Avidan L, Ofir D, Melayev A, Kalichman L. The effect of additional ankle and midfoot mobilizations on plantar fasciitis: a randomized controlled trial. J Orthop Sports Phys Ther. 2015;45(4):265-72.

41. Snodgrass SJ, Rivett DA, Sterling M, Vicenzino B. Dose optimization for spinal treatment effectiveness: a randomized controlled trial investigating the effects of high and low mobilization forces in patients with neck pain. J Orthop Sports Phys Ther. 2014;44(3):141-52.

42. Farooq MN, Mohseni-Bandpei MA, Gilani SA, Ashfaq M, Mahmood Q. The effects of neck mobilization in patients with chronic neck pain: A randomized controlled trial. J Bodyw Mov Ther. 2018;22(1):24-31

43. Mayor EE, Pérez GL, Martín YP, del Barco ÁA, Fuertes RR, Requejo CS, et al. Ensayo clínico aleatorizado en pacientes con cervicalgia mecánica en atención primaria: terapia manual frente a electroestimulación nerviosa transcutánea. Aten Primaria. 2008:40(7):337-43.

44. Villafañe JH, Silva GB, Fernandez-Carnero J. Effect of thumb joint mobilization on pressure pain threshold in elderly patients with thumb carpometacarpal osteoarthritis. J Manipulative Physiol Ther. 2012;35(2):110-20.

45. Villafañe JH, Langford D, Alguacil-Diego IM, Fernández-Carnero J. Management of trapeziometacarpal osteoarthritis pain and dysfunction using mobilization with movement technique in combination with kinesiology tape: a case report. J Chir Med. 2013;12(2):79-86.

46. Villafañe JH, Silva GB, Diaz-Parreño SA, Fernandez-Carnero J. Hypoalgesic and motor effects of kaltenborn mobilization on elderly patients with secondary thumb carpometacarpal osteoarthritis: a randomized controlled trial. J Manipulative Physiol Ther. 2011;34(8):547-56 
47. Villafañe JH, Cleland JA, Fernandez-De-Las-Peñas C. Bilateral sensory effects of unilateral passive accessory mobilization in patients with thumb carpometacarpal osteoarthritis. J Manipulative Physiol Ther. 2013;36(4):232-7.

48. Horst R, Maicki T, Trąbka R, Albrecht S, Schmidt K, Mętel S, et al. Activity-vs. structural-oriented treatment approach for frozen shoulder: a randomized controlled trial. Clin Rehabil. 2017;31(5):686-95.

49. Sterling M, Pedler A, Chan C, Puglisi M, Vuvan V, Vicenzino B. Cervical lateral glide increases nociceptive flexion reflex threshold but not pressure or thermal pain thresholds in chronic whiplash associated disorders: a pilot randomised controlled trial. Man Ther. 2010;15(2):149-53.

50. Hidalgo B, Hall T, Bossert J, Dugeny A, Cagnie B, Pitance L. The efficacy of manual therapy and exercise for treating non-specific neck pain: A systematic review. J Back Musculoskelet Rehabil. 2017;30(6):1149-69.

51. Hidalgo B, Detrembleur C, Hall T, Mahaudens P, Nielens H. The efficacy of manual therapy and exercise for different stages of non-specific low back pain: an update of systematic reviews. J Man Manip Ther. 2014;22(2):59-74.
52. Jayaseelan DJ, Scalzitti DA, Palmer G, Immerman A, Courtney CA. The effects of joint mobilization on individuals with patellofemoral pain: a systematic review. Clin Rehabil. 2018;32(6):722-33.

53. Haik M, Alburquerque-Sendín F, Moreira R, Pires E, Camargo P. Effectiveness of physical therapy treatment of clearly defined subacromial pain: a systematic review of randomised controlled trials. Br J Sports Med. 2016:bjsports-2015-095771.

54. Stathopoulos N, Dimitriadis Z, Koumantakis GA. Effectiveness of Mulligan's Mobilization with Movement techniques on pain and disability of peripheral joints: A systematic review with meta-analysis between 2008-2017. Physiotherapy. 2018.

55. Gross A, Kay TM, Paquin JP, Blanchette S, Lalonde P, Christie T, et al. Exercises for mechanical neck disorders. Cochrane Database Syst Rev. 2015;1:CD004250.

56. May M, Junghaenel DU, Ono M, Stone AA, Schneider S. Ecological Momentary Assessment Methodology in Chronic Pain Research: A Systematic Review. J Pain. 2018;19(7):699-716.

57. Swait G, Finch R. What are the risks of manual treatment of the spine? A scoping review for clinicians. Chiropr Man Therap. 2017;25:37. 